Fast and robust hydrogen sensors based on discontinuous palladium films on polyimide, fabricated on a wafer scale

This article has been downloaded from IOPscience. Please scroll down to see the full text article.

2010 Nanotechnology 21505501

(http://iopscience.iop.org/0957-4484/21/50/505501)

View the table of contents for this issue, or go to the journal homepage for more

Download details:

IP Address: 128.178.195.80

The article was downloaded on $24 / 11 / 2010$ at $14: 00$

Please note that terms and conditions apply. 


\title{
Fast and robust hydrogen sensors based on discontinuous palladium films on polyimide, fabricated on a wafer scale
}

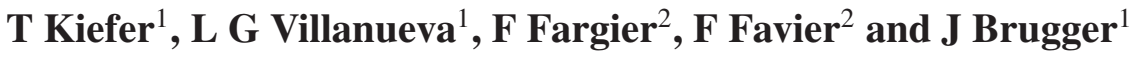 \\ ${ }^{1}$ Microsystems Laboratory, Station 17, École Polytéchnique Fédérale de Lausanne (EPFL), \\ CH-1015 Lausanne, Switzerland \\ ${ }^{2}$ Institut Charles Gerhardt, AIME, UMR 5253 CNRS, Université Montpellier 2, cc015, \\ F-34095 Montpellier, Cedex 05, France \\ E-mail: juergen.brugger@epfl.ch
}

Received 17 May 2010, in final form 28 October 2010

Published 23 November 2010

Online at stacks.iop.org/Nano/21/505501

\begin{abstract}
Fast hydrogen sensors based on discontinuous palladium (Pd) films on supporting polyimide layers, fabricated by a cost-efficient and full-wafer compatible process, are presented. The films, deposited by electron-beam evaporation with a nominal thickness of $1.5 \mathrm{~nm}$, consist of isolated Pd islands that are separated by nanoscopic gaps. On hydrogenation, the volume expansion of Pd brings initially separated islands into contact which leads to the creation of new electrical pathways through the film. The supporting polyimide layer provides both sufficient elasticity for the Pd nanoclusters to expand on hydrogenation and a sufficiently high surface energy for good adhesion of both film and contacting electrodes. The novel order of the fabrication processes involves a dicing step prior to the Pd deposition and stencil lithography for the patterning of microelectrodes. This allows us to preserve the as-deposited film properties. The devices work at room temperature, show response times of a few seconds and have a low power consumption of some tens of $\mathrm{nW}$.

S. Online supplementary data available from stacks.iop.org/Nano/21/505501/mmedia
\end{abstract}

(Some figures in this article are in colour only in the electronic version)

\section{Introduction}

Hydrogen $\left(\mathrm{H}_{2}\right)$ has raised considerable interest as a future energy carrier in its application to fuel-cell-driven vehicles, as it is absolutely free of polluting emissions. Because it is flammable between concentrations of $4 \%$ and $75 \%$ in air, safety sensors for leak detection are required. In recent years, a variety of sensor concepts has emerged such as resistivitybased [1, 2], MOS-based [3, 4], optical [5], cantilever-based [6] or nanogap-based sensors [7,8]. Most of these devices make use of palladium $(\mathrm{Pd})$ as the sensitive material because of its highly catalytic surface and a large solubility of atomic hydrogen $[9,10]$. To pave the way for commercial usage, such sensors need not only to be fast and sensitive below the lower explosion limit (LEL) of hydrogen in air, but also robust, reliable and manufacturable on large scales at low costs.

It has been shown that the use of ultrathin discontinuous Pd films [11] offers a simple solution to realize a hydrogen sensor that could fulfill many of those requirements. Discontinuous films are commonly deposited by physical vapor deposition (PVD) techniques such as electron-beam evaporation. Such films, being at an early stage of film growth, exhibit an island-like disconnected morphological structure and inherently include a multitude of nanoscopic gaps [12]. Inter-island distances are usually found to be in the range of a few nanometers. By exposing a discontinuous Pd film to hydrogen, the volume expansion of Pd $[9,10]$ leads to mechanical narrowing or even closing of the nanogaps, giving rise to the formation of new electrical pathways. This results in a decrease of the sensor resistance. Advantages of nanocrystalline films for hydrogen sensing are an increased solubility of hydrogen [13, 14], a larger volume increase [15-17] and less hysteresis [18] when compared with bulk palladium. Deposited on rigid substrates such as $\mathrm{SiO}_{2}$, discontinuous $\mathrm{Pd}$ films showed poor hydrogen sensing 
a)

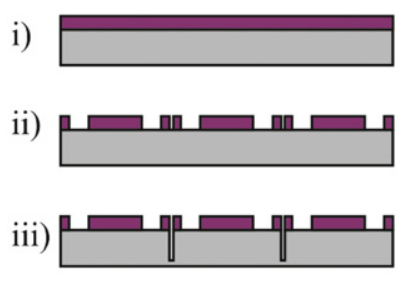

iv)

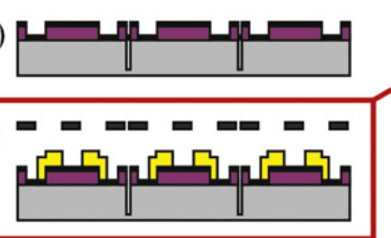

vi)

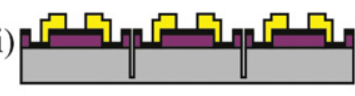

vii)

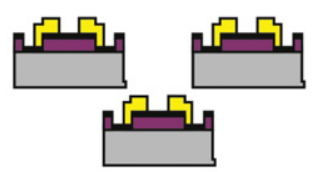

viii)

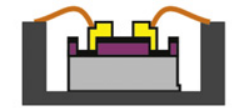

b)
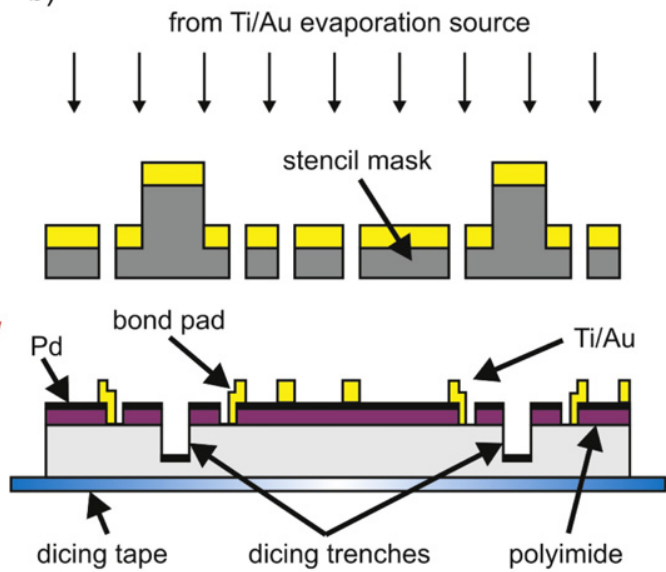

Figure 1. (a) Process flow for sensor fabrication and (b) detailed schematic representation of process step (v) which is the deposition of electrodes on the pre-diced wafer using stencil lithography.

results, due to an insufficient in-plane relaxation of mechanical stress which is built up during hydrogen absorption inside the Pd islands [19]. Xu et al [11] showed highly improved sensing characteristics and fast response times by the use of an intermediate silane-based self-assembled monolayer (SAM). Here, the lower surface free energy of the SAM increased the surface mobility of the Pd clusters.

However, the use of low surface energy substrates might entail poor adhesion of both the Pd film and connecting electrodes which could compromise the mechanical reliability of the devices in their application. We have previously shown that thin polymer films such as polyimide (PI) allow a palladium film to expand sufficiently for hydrogen sensing, while maintaining good adhesion properties [20]. Further, PI shows high chemical inertness, temperature stability up to several hundred degrees [21] and can easily be deposited by standard spin-coating processes. This makes it also interesting as a supporting layer for discontinuous Pd films.

In the present work we demonstrate a hydrogen sensor based on discontinuous palladium films on a supporting polyimide layer. The devices are manufacturable using simple and common microfabrication techniques and show a fast response to hydrogen well below the lower explosion limit of $\mathrm{H}_{2}$ in air, low power consumption in the $\mathrm{nW}$ range and good mechanical and temperature stability. The simple fabrication methods used and hence low manufacturing costs show a high potential for industrial purposes.

\section{Fabrication}

The sensor fabrication includes three main steps: (i) the deposition of the supporting polymer layer, (ii) the unmasked evaporation of the sensitive palladium film and (iii) the stencilassisted deposition of the microelectrodes. To ensure that the film properties are preserved to a maximum extent, stencil lithography [22], a resistless deposition technique based on evaporation and shadow masking, is used to deposit the electrodes. To enable full-wafer compatible processing, fullwafer bulk silicon stencil masks are used which further provide good mechanical stability for handling (for further information on stencil fabrication, see supplementary information available at stacks.iop.org/Nano/21/505501/mmedia).

\subsection{Process flow for sensor fabrication}

The process flow for sensor fabrication is shown in figure 1(a). It starts with the deposition of the supporting PI film on a standard silicon wafer by spin-coating (step (i)). Large openings in the polymer layer can be defined by photolithography if the final gold wire bonding process after packaging requires a good mechanical strength of the bonds (step (ii)). Since metal adhesion on polymer surfaces is usually rather poor, the connecting metal electrodes are deposited across the structured polymer and the wire bonding can be carried out on top of the solid wafer surface. A wafer dicing process is performed prior to the palladium evaporation which separates the wafer into individual chips. This process order ensures that the fragile film is not exposed to either the cooling water jet that is used during dicing, or to mechanical vibrations that occur (step (iii)). A remaining thickness of $50 \mu \mathrm{m}$ is being left in the silicon since complete cutting would cause the dicing tape to relax. This would change the distance between the separated chips and impede the subsequent alignment of the stencil mask that includes the electrode design. The deposition of the Pd films is carried out using electron-beam evaporation at room temperature and an evaporation rate of $0.1 \AA \mathrm{s}^{-1}$ (step (iv)). A low ambient pressure of $5 \times 10^{-7}$ mbar is used in order to avoid excessive deposition of atmospheric residuals that can become significant for low evaporation rates [23]. The effective thickness of the films, as measured by a quartz balance, is $1.5 \mathrm{~nm}$.

Now, the stencil mask is aligned to the wafer which still sticks to the dicing tape, and Ti/Al electrodes with various electrode spacings between 50 and $1000 \mu \mathrm{m}$ are deposited (step (v) with details in figure 1(b)). Post-deposition of the electrodes makes sure that a good electrical contact to the discontinuous film is obtained and that no inhomogeneities in film properties arise at the electrode-film interface [24]. Figure 2(a) shows the pre-diced wafer and the stencil mask 
a)

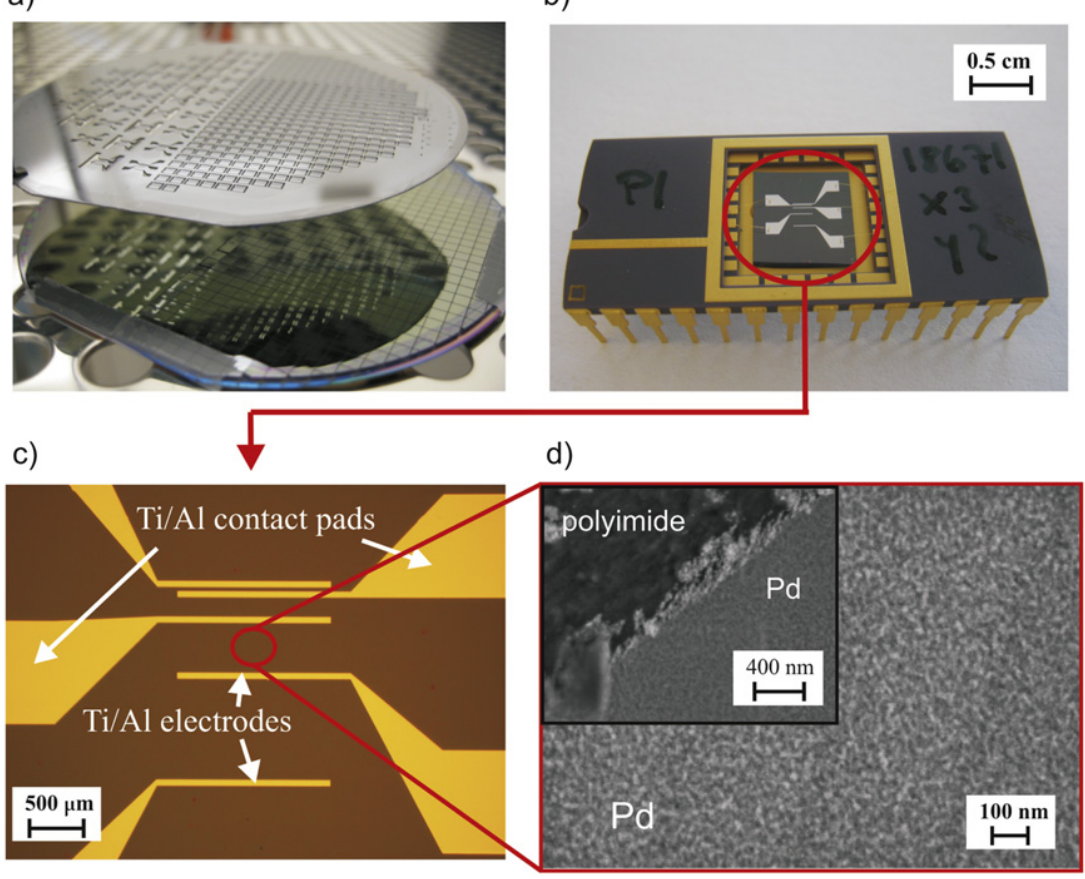

Figure 2. (a) Optical image of a stencil mask on top of a pre-diced wafer, (b) a wire-bonded sensor in a DIL package, (c) optical image of the contacting electrodes and (d) SEM image of the nominally $1.5 \mathrm{~nm}$ thin Pd film on polyimide and a blank polyimide surface that was masked during Pd deposition (inset image).

before alignment and deposition. Subsequently, the stencil is removed (step (vi)), the chips are separated (step (vii)) and wire-bonded into standard dual in-line ceramic packages (step (viii)). An image of the final sensor is shown in figure 2(b), with a close-up optical image of the electrode region in figure $2(\mathrm{c})$.

\section{Results and discussion}

\subsection{Pd film properties}

An SEM image of the $1.5 \mathrm{~nm}$ thick Pd film on a $2 \mu \mathrm{m}$ thin polyimide layer is displayed in figure 2(d). The inset in the top left corner shows the difference in contrast between the blank PI surface and the additional Pd film. The Pd film consists of homogeneously distributed nanoclusters, roughly estimated to be around $10 \mathrm{~nm}$ in size by means of the SEM image. The significant surface roughness on the polyimide film did not permit AFM measurements that could resolve the contrast in the deposited Pd clusters. For DC bias voltages up to several volts, linear $I V$ characteristics were obtained. This can be understood under the assumption of a multitude of tunnel junctions between the metal islands where a high external potential drop breaks up into small potential drops across each individual junction $[25,26]$. An individual junction will then still be in the so-called low bias regime, where approximately linear IV behavior is obtained [27].

The relatively high surface free energy of the PI surface provides good adhesion properties of the $\mathrm{Pd}$ film. Ultrasonication at various frequencies $f(f=$ $25 \mathrm{kHz} / 45 \mathrm{kHz} / 1 \mathrm{MHz}$ ) up to 40 min were carried out and the electrical resistance of the films was measured. No significant changes before and after the treatment were observed, indicating that neither the Pd film nor the metal electrodes suffered damage. Similar experiments were performed on sensors using a self-assembled monolayer (Trichloro( $1 H, 1 H$, $2 \mathrm{H}, 2 \mathrm{H}$-perfluorooctyl)silane) as a supporting layer. $\mathrm{Xu}$ et al [11] pointed out that a low surface free energy is advantageous for the ability of the Pd clusters to expand during hydrogen exposure. However, ultrasonication resulted in severe delamination of both the Pd film and the electrodes on our SAM layers, which made the sensors unusable.

Further, thermal annealing for a duration of $60 \mathrm{~min}$ and temperatures $T$ increasing from $T=30$ up to $200^{\circ} \mathrm{C}$ was carried out in an oven under atmospheric conditions. No significant change in electric film resistance occurred up to temperatures of $150{ }^{\circ} \mathrm{C}$. Above this, the film resistance increased rapidly, which we attribute to coalescence of the metal clusters. Initially separated islands start to agglomerate and the average inter-island distance within the film increases [19].

Temperature measurements in air revealed a negative temperature coefficient of resistance (TCR) which is typical for discontinuous metal films [28]. Together with electrical resistances in the $\mathrm{M} \Omega$ range, it suggests a disconnected islandlike morphological film structure. Therein, electric transport mechanisms such as thermally activated quantum tunneling between neighboring islands are likely to govern the film characteristics [12].

\subsection{Characterization methods}

Electrical measurements of the sensors in hydrogen were carried out using a sealed gas flow cell made out of 


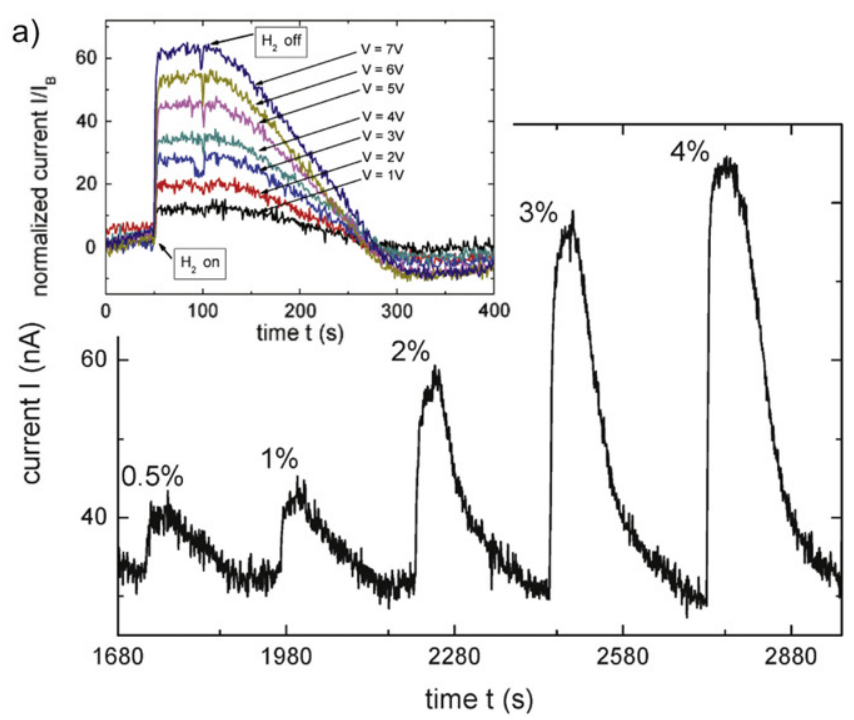

b)

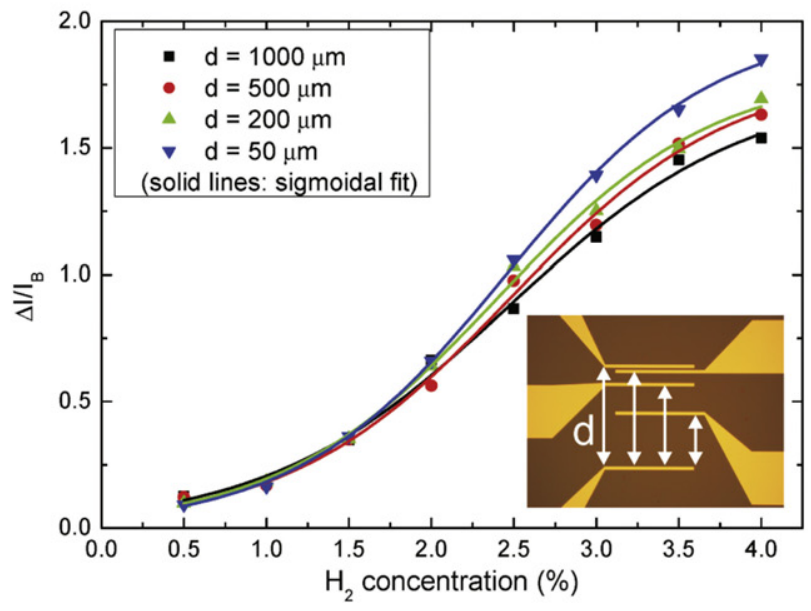

Figure 3. (a) Representative sensor response to various concentrations $(V=500 \mathrm{mV})$ and (b) relative change in current as a function of $\mathrm{H}_{2}$ concentration for various electrode spacings $d$.

polymethylmethacrylate (PMMA) comprising an inlet for the hydrogen/nitrogen gas mixture and an outlet. Thereto, the sensor packages were mounted onto a printed circuit board (PCB) with electrical connections and placed inside the cell. A constant electric potential $V$ was applied and the current $I$ was measured by a picoampere meter. The hydrogen and nitrogen gas flow rates were controlled by calibrated mass flow meters and a flow controller (Vögtlin Instruments AG). In order to exclude cross-sensitivities caused by the $\mathrm{N}_{2}$ carrier gas as well as the velocity of the gas flow, the devices were tested with pure $\mathrm{N}_{2}$ and mixed $\mathrm{H}_{2} / \mathrm{N}_{2}$ flows at various flow rates between 25 and $200 \mathrm{ml} \mathrm{min}{ }^{-1}$. No changes in the electronic signals were observed.

\subsection{Hydrogen sensing}

Exposure of the sensors to $\mathrm{H}_{2} / \mathrm{N}_{2}$ mixtures resulted in an instantaneous, sharp increase in electric current $\Delta I$ which saturated after a few seconds (see figure 3(a)). It showed a pronounced dependence on the $\mathrm{H}_{2}$ concentration between concentrations of $0.5 \%$ and $4 \% \mathrm{H}_{2}$. The recovery time was in the range of some tens of seconds. No bias dependence on the sensor kinetics has been observed for moderate electrical potentials, here $1 \mathrm{~V}<V<7 \mathrm{~V}$ (see the inset of figure 3(a)). As has likewise been reported for devices on other substrate materials such as glass, SiN or SAMs, we associate the decrease in electrical resistance that is observed with changes in inter-island distances and eventually mechanical closing of inter-island gaps. This densification of the film by the volume expansion of the Pd clusters triggers the formation of new electrical pathways that percolate through the film. The power consumption of the devices in air is in the range of some few tens of $\mathrm{nW}$, which is related to the high electrical resistance.

Cyclic $\mathrm{H}_{2}$ /air exposures of the sensors revealed that the $\mathrm{Pd}$ film resistance is subject to irreversible changes. They appear as a reduction of current change $\Delta I$ with the number of cycles, following an exponential decrease of $\Delta I$ with

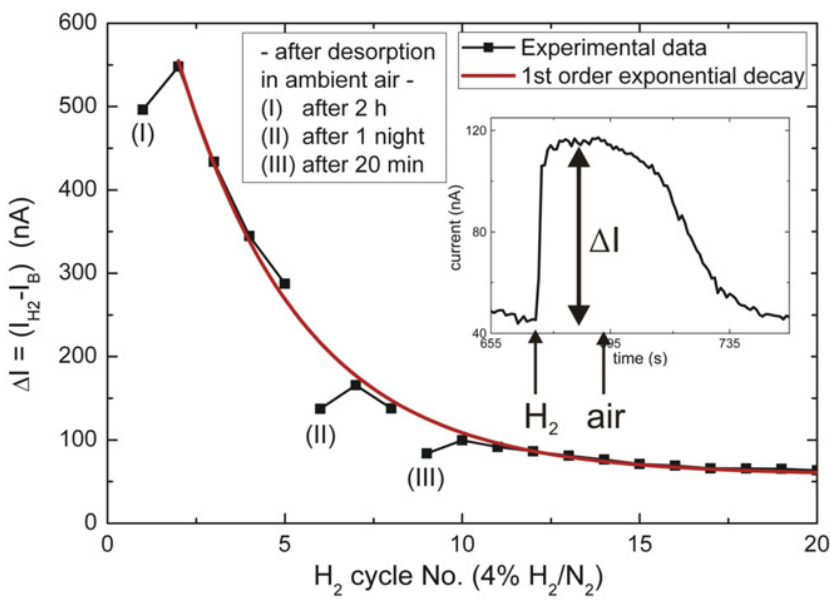

Figure 4. Priming effect, i.e. decrease of signal change at hydrogen exposure with number of $\mathrm{H}_{2}$ /air cycles (here, 20 cycles) and effect of exposure to ambient air. $(V=1 \mathrm{~V})$.

increasing numbers of cycles (see figure 4 for the result of cyclic exposures to $4 \% \mathrm{H}_{2}$ ). The presence of such priming effects can, for instance, be the result of mechanical reorganization of the nanoclusters or breaking of connections between initially connected islands caused by the lattice dilatation and contraction [7]. Further, uptake of hydrogen in deep energy traps at grain boundaries may cause irreversible strain in the material [29]. Reproducible measurements can hence only be carried out when no further drift of the sensor response is guaranteed. For the present sensors this was the case after approximately 20 cycles.

Deviations from the exponential trend in signal decrease are observed when the sensor is exposed to ambient air for a longer time period. The hydrogen response following a longer exposure to air (see data points I, II and III in figure 4 for the first, sixth and eighth cycle, respectively) shows a reduced $\Delta I$ as compared to what is predicted by the trend curve. Since 
subsequent cycles fall back on the original exponential trend we associate this observation by two main effects: (i) adsorbed oxygen on the palladium surface that is reduced by hydrogen molecules to form water and (ii) the presence of a thin water film in air which desorbs under the influence of the dry $\mathrm{H}_{2} / \mathrm{N}_{2}$ gas flow. After leaving the sensor under a constant $\mathrm{N}_{2}$ flow during $12 \mathrm{~h}$, this effect does not occur, which confirms the hypothesis.

Figure 3(b) shows concentration measurements for various electrode spacings $d$. All the plots follow a sigmoidal shape fit, suggesting that percolation is an important factor in the electronic transport process [8, 30]. However, a change in geometrical dimensions of the film by choosing electrodes with different spacings $d$ does not reveal any significant dependence. According to a recent theoretical study from our group on percolation in rectangular domains, only minor changes in conduction behavior can be expected by such geometrical changes [30] which indicates that our experimental findings could in fact be anticipated. Yet, with the choice of closer electrode spacings an overall decrease in electrical resistance can be obtained while similar sensing performances are maintained. This in turn allows the use of relatively simple electronic equipment for signal readout, which is highly desirable for integrated sensor applications.

\subsection{Conclusions}

Discontinuous palladium (Pd) films, created by electron-beam evaporation on supportive polyimide layers, were used for the cost-efficient fabrication of fast and robust hydrogen sensors. The dedicated process order and the use of stencil lithography allowed for full-wafer fabrication while preserving the asdeposited film properties. Thereafter, chip separation by dicing is performed before the film deposition and stencil lithography is used to deposit Ti/Al electrodes on top. The polyimide layer ensured both very good mechanical stability of the Pd films and electrodes, and the necessary flexibility for in-plane relaxation of hydrogen-induced stress in the Pd nanoclusters. The films withstand ultrasonication and temperatures up to $150{ }^{\circ} \mathrm{C}$ without significant changes in electrical resistance. The devices work at room temperature and detect hydrogen well below hydrogen concentrations of $4 \%$ within a few seconds and a power consumption of some tens of nW. Priming the sensor by alternating hydrogen/air cycles showed improvement in the reproducibility of the sensor response.

\section{Acknowledgments}

The authors thank the FP6 Integrated Project HySYS SES6019981 for financial support, the Center of MicroNanofabrication $(\mathrm{CMI})$ and the Interdisciplinary Center for Electron Microscopy (CIME) at EPFL in Lausanne for SEM imaging,
Dr Michel Rossi for the kind supply of equipment for the hydrogen measurement set-up and Sebastian Haller for preliminary thin film characterization.

\section{References}

[1] Cabrera A L and Aguayo-Soto R 1997 Catal. Lett. 4579

[2] Offermans P, Tong H D, van Rijn C J M, Merken P, Brongersma S H and Crego-Calama M 2009 Appl. Phys. Lett. 94223110

[3] Lundström I, Shivaraman M S and Svensson C 1977 Surf. Sci. 64497

[4] Dwivedi D and Srivastava S K 2000 Sensors Actuators B 71161

[5] Zhao Z, Carpenter M, Xia H and Welch D 2006 Sensors Actuators B 113532

[6] Baselt D R, Fruhberger B, Klaassen E, Cemalovic S, Britton C L Jr, Patel S V, Mlsna T E, McCorkle D and Warmack B 2003 Sensors Actuators B 88120

[7] Favier F, Walter E C, Zach M P, Benter T and Penner R M 2001 Science 2932227

[8] Walter E C, Favier F and Penner R M 2002 Anal. Chem. 741546

[9] Lewis F A 1976 The Palladium Hydrogen System (New York: Academic)

[10] Flanagan T B and Oates W A 1991 Annu. Rev. Mater. Sci. 21269

[11] Xu T, Zach M P, Xiao Z L, Rosenmann D, Welp U, Kwok W K and Crabtree G W 2005 Appl. Phys. Lett. 86203104

[12] Morris J R and Coutts T J 1977 Thin Solid Films 473

[13] Mütschele T and Kirchheim R 1987 Scr. Metall. 21135

[14] Lemier C and Weissmüller J 2007 Acta Mater. 551241

[15] Weissmüller J and Lemier C 1999 Phys. Rev. Lett. 82213

[16] Suleimann M, Jisrawi N M, Dankert O, Reetz M T, Bähtz C, Kirchheim R and Pundt A 2003 J. Alloys Compounds 644 356

[17] Ingham B et al 2008 Phys. Rev. B 78245408

[18] Pundt A and Kirchheim R 2006 Rev. Meter. Res. 36555

[19] Dankert O and Pundt A 2002 Appl. Phys. Lett. 811618

[20] Kiefer T, Favier F, Vazquez O, Villanueva G and Brugger J 2008 Nanotechnology 19125502

[21] Androva N A, Bessonov M I, Laius L A and Rudakov A P 1970 Polyimides, A New Class of Thermally Stable Polymers (Stamford: Technomic Publications)

[22] Brugger J, Berenschot J, Kuiper S, Nijdam W, Otter B and Elwenspoek M 2000 Microelectron. Eng. 53403

[23] Mahan J E 2000 Physical Vapor Deposition of Thin Films (New York: Wiley)

[24] Borziak P, Dyukov V, Kostenko A, Kulyupin Y and Nepijko S 1976 Thin Solid Films 3621

[25] Hill R M 1969 Proc. R. Soc. A 309397

[26] Neugenbauer C A and Webb M B 1962 J. Appl. Phys. 2274

[27] Simmons J G 1963 J. Appl. Phys. 34238

[28] Campbell D S and Morley A R 1971 Rep. Prog. Phys. 34283

[29] Stuhr U, Wipf H, Udovic T J, Weissmüller J and Gleiter H 1995 J. Phys.: Condens. Matter 7219

[30] Kiefer T, Villanueva G and Brugger J 2009 Phys. Rev. E. 80021104 